\title{
Cultural and Religious Belief Approaches of a Tuberculosis Program for Hard-to-Reach Populations in Mentawai and Solok, West Sumatera, Indonesia
}

\author{
Rizanda Machmud ${ }^{1 *}$, Irvan Medison², Finny Fitry Yani ${ }^{3}$
}

\author{
${ }^{1}$ Community Medicine and Public Health Department, Faculty of Medicine, Universitas Andalas, Indonesia \\ 2Pulmonary Department, Faculty of Medicine, Universitas Andalas, Indonesia \\ ${ }^{3}$ Child Department, Faculty of Medicine, Universitas Andalas, Indonesia
}

\begin{abstract}
Tuberculosis (TB) is a leading public health concern in Indonesia. It ranks second on the list of high-burden TB countries. In West Sumatra, 47\% of TB cases are undetected, late-diagnosed, and received incomplete treatment because of low-level awareness and knowledge and stigma, especially among the hardest to reach populations. The study aimed to identify the best communication channel to reach those who live in vulnerable and remote areas. This study was a qualitative study applying in-depth interviews to the informal leaders, health officers, cultural artists, and religious leaders across districts in Mentawai and Solok Districts, which are remote and had the lowest case detection rates compared with other districts. The questionnaire was prepared with the perception of the channel to identify TB cases. The data were analyzed using the content analysis technique. Involving religious and informal leaders and using traditional music as a communication channel improved the population's awareness of TB symptoms and access to TB testing and treatment, as well as reduced TB-related stigma. This study found that the cultural and religious contexts play a major role in health communication on TB control for hard-to-reach populations in West Sumatera, Indonesia.
\end{abstract}

Keywords: channel, religious belief, stigma, traditional party, tuberculosis

\section{Introduction}

Tuberculosis (TB) infection remains one of the biggest health problems in Indonesia, which ranked second in the world on the list of countries with a high burden of TB. ${ }^{1,2}$ In many low- and middle-income countries with high TB burdens, the government take necessary action to stop TB spreading. ${ }^{1}$ Case detection rates (CDRs) in the Indonesian National Tuberculosis Program have remained steady or have slightly declined recently under notification and access barriers to diagnostic facilities. ${ }^{2}$

Tuberculosis control problems are associated with a low level of awareness and knowledge of TB, mainly because of the community's high level of TB stigma. $3-5$ Stigma in Indonesia is one of the major burdens that cause patients to be antisocial. The reasons underlying this behavior is mainly related to the belief that TB is a hereditary disease, a magic-related disease, or a disease of indigent people. Therefore, it is shameful to have a family member suffering from TB. ${ }^{1,5}$ The stigma of infectious diseases, including $\mathrm{TB}$, is usually observed among isolated communities and in populations of low

Correspondence * Rizanda Machmud, Community Medicine and Public Health Department, Faculty of Medicine, Universitas Andalas, Indonesia, Komplek Unand Limau Manis, Padang, Sumatera Barat, Indonesia, Phone: +62 812662 3467,Email: rizanda_machmud@yahoo.com education and socioeconomic status, many of whom are not reached by the healthcare system. ${ }^{3-5}$ On the other hand, TB is typically under detected among hard-to-reach populations, including people living in urban slums, postdisaster areas, and remote areas; the poor; contacts of TB patients; people living with HIV/AIDS; and malnourished individuals. ${ }^{6-9}$

As the largest archipelago country globally, Indonesia is $80 \%$ water and only $20 \%$ of the land, covering 2 million $\mathrm{km}^{2}$. Most of the 235 million Indonesian people are spread over five main islands (Sumatra, Java, Kalimantan, Sulawesi, and Irian Jaya). The remaining individuals live on about 13,000 smaller islands, many of which are isolated. Solok and Mentawai Districts are located on the western part of Sumatera Island and are quite remote and in an earthquake-prone area. In both areas, the TB CDRs are lower than those in other districts when the study was initially implemented in 2016. These areas also have lower Human Development Index scores. The Solok District ranks $14^{\text {th }}$ out of 19 , and the Mentawai District ranks the lowest. ${ }^{1}$ Intensified efforts are required to reduce $\mathrm{TB}$ transmission and accelerate

Received : October 21, 2019

Accepted : August 14, 2020

Published: November 28, 2020 
reductions in TB incidence, particularly in this challenging terrain and among these hard-to-reach populations.

Over the past few years, public health promotion efforts have tried to engage faith-based organizations to reach target populations effectively. ${ }^{10}$ The involvement of religious leaders in health-related interventions has generally improved their congregations' participation and promoted positive health outcomes. ${ }^{10}$ Religious and spiritual leaders are some of the most credible people who influence people of faith's thoughts, emotions, and actions. ${ }^{11}$ People of faith often make a conscious decision to behave in a manner that is consistent with the expectations of their religious or spiritual messages. ${ }^{12,13}$

Study in another setting has shown the need to address cultural health beliefs about the locus of control in designing and developing TB care and treatment programs. ${ }^{10,12}$ The study results revealed that foreign-born Vietnamese clients tended to operate with an internal locus of control, to attribute the resolution of health problems, such as TB, to chance rather than something that could be controlled by others. A controlled study of an HIV/AIDS/STI/TB intervention with faith healers in the Vhembe District, South Africa, also mentions faith healers' role. They could be more widely utilized in HIV/AIDS/STI/TB prevention programs as risk reduction counselors, in particular, to advise on matters of community-level education. ${ }^{15}$

Many research studies have reported that a cultural approach can also be a channel to deliver edutainment (educational entertainment). ${ }^{13}$ People in West Sumatera are mostly of the Minang tribe and have culturally-rooted health beliefs and practices. There is a folk tradition of the Minangkabau ethnic group, Saluang, which incorporates music, singing, and storytelling. It is very popular and is usually performed for traditional ceremonies and festivals. Stories are delivered by both acting and singing and are mostly based on Minangkabau legends and folktales. It assumes that every ethnicity is influenced explicitly by its cultural background and requires a different health communication channel to be delivered to fulfill its needs. Many research studies have reported that a cultural approach can be a channel to deliver edutainment (educational entertainment) about TB. ${ }^{13}$

This study was designed to identify cultural and religious belief approaches for the hard-to-reach population in the Tuberculosis Program and expand communitybased models to achieve universal access to TB testing and treatment in Mentawai and Solok, West SumateraIndonesia.

\section{Method}

This study was a qualitative study using purposive sampling in selecting officers, informal leaders, and cadre members were eligible to participate in the study. The number of interviews was guided by reaching data saturation. Data saturation was finally reached 64 informants.

This study conducted in-depth semi-structured interviews. Informants were directly contacted and informed of the purpose of the study. All face-to-face interviews took place at a date and time that was most convenient for participants. Interviewers asked open-ended questions to obtain perceptions about methods that might be best used to increase community knowledge and awareness of TB programs, thus increasing CDRs. The interviewer used probing questions to clarify a situation or to obtain detail answer, such as what kind of experience that they gain in TB control, if there is challenge, how they tackled the problems. For further questions, exploring the best channel that could be a suitable approach in delivering Tuberculosis Program for hard-to-reach populations, it refer to occasion that people mostly come and gather. In-field notes were taken to cover untold aspects.

All interviews were recorded and immediately transcribed verbatim. The transcripts were read by the authors several times to get insight into the participants' experiences. After that, they were analyzed using conventional content analysis according to the technique. Data were coded and related codes were finally grouped under specific categories using the constant comparative analysis. After each new interview, the previous categories were either revised or combined. Otherwise, a new category was created. All authors contributed to category development through face-to-face meetings.

The transcripts were thematically analyzed. The analysts coded the transcripts and reviewed, discussed, and refined the coding schemes until consensus was reached. The derivation of themes shown in Table 1 illustrates the data analysis, questions, meaning units of answers, codes, categories, and sub-themes.

Emerging concepts were assessed using the constant comparative method from grounded theory. This study developed and expanded community-based models for achieving universal access to TB testing and treatment.

\section{Results}

Sixty-four informants completed an in-depth interview and focus group discussion in Solok and Mentawai Districts with the lead researcher. Table 1 describes limited characteristics to protect anonymity. The informants' academies included a varying range of schools, including primary, secondary, faith, and music schools. The informants' ages varied between 19 and 65 years. Most cultural artists, officers, and cadres were middleaged. All cadres were females, and the remaining informants were males.

Knowledge, Attitudes, and Practices (KAP) and 


\section{Awareness of TB}

According to the informants, almost all officers, informal leaders, and cadre members perceived KAP and awareness towards TB were low, affecting TB coverage to remains low because TB is still stigmatized and knowledge about the disease and its treatment remains low. Statements from cadre members indicated that community members still believe that TB is a hereditary disease, is shameful for family members of affected people, is a disease of indigent people, and is a magic-related disease.

They said that the cough is caused by the magic/spell

from someone (TB volunteer, 35).

There is also a stubborn suspect, and they do not want to get the test. They said [that] Chinese medicine is more effective for them. One of my TB suspects already died before getting a test (TB Volunteer 40).

Another comment from TB volunteers due to the stigma of TB.

Sometimes a TB suspect hides the symptoms because they feel ashamed, and the patient who got $T B$ does not want other people to know that they got TB. They are afraid when people know (TB volunteer, 49).

This TB stigma can harm an individual and may cause a delay in the person seeking treatment. The TB officers in both the Mentawai and Solok Districts mentioned that CDRs in the National Tuberculosis Program has recently remained steady or have slightly declined because of under notification and barriers to accessing diagnostic facilities. One informant, a medical doctor who provides primary healthcare in both the Mentawai and Solok Districts, also mentioned that TB is a disease with many elusive patients. He meant that most patients are asymptomatic or have mild symptoms that are not clinically apparent. The healthcare provider realized that only a small fraction of patients with TB were being diagnosed and treated. Due to the diversity in TB's clinical manifestations, not all patients in the community are being identified by healthcare providers. In general, only patients with severe manifestations are likely identified. Because of this situation, CDRs are lower than the target program.

\section{The System of TB Control in the Community}

The informal leaders and cadre members mentioned their perceptions that there was no comprehensive approach. Also, there was no coordination to engage the community in supporting TB services, including helping patients stay on treatment, proactively identifying possible TB cases, and advocating for better quality TB services. The TB officers realized that community systems had limited capacity to increase TB's public knowledge or support improved TB diagnosis and treatment. It seems that the system of TB control in the community has not been planned optimally.

\section{Hard-to-Reach Populations}

Both districts in this study are hard-to-reach populations. The Mentawai District consists of a cluster of 99 large and small islands surrounded by the Indian Ocean. Most residents live on four main islands: Siberut, Sipora, North Pagai, and South Pagai. Boats are the only transportation between the islands. On the other hand, the Solok District's topography varies greatly between plains, valleys, rolling hills, and mountains ranging from 329 to 1,458 meters above sea level.

The TB officers in both districts mentioned bear geographic burdens in their areas. They must traverse broad and remote areas to reach TB patients. The TB officers and cadre members mentioned that they have to travel by foot for over two hours to visit the outreach community because there are no roads. Sometimes landslides and storms isolate some areas. In both the Mentawai and Solok Districts, many people live in urban slums, on remote islands, or in post-disaster areas. These individuals are poor and sometimes malnourished and have contacts with TB patients. These two districts were selected because they are post-disaster areas where TB CDRs are low compared to the other districts.

The present study found that different health communication channels were needed in different regions based on their different cultures and beliefs. People in the Mentawai District speak various languages, and many in the community practice different religions. The TB officer and medical doctor were challenged in health communication because more than one language are spoken in the

Table 1. Informants Characteristics

\begin{tabular}{lccclc}
\hline Proffesion & Solok & Mentawai & Age & Education & Sex \\
\hline Officer & 2 & 2 & 30-40 years old & Bachelor & All Males \\
Informal leader & 9 & 8 & 52-65 years old & Senior high school-bachelor & All Males \\
Cadre & 12 & 11 & 19-36 years old & Junior-senior high school & All Females \\
Religious leader & 7 & 6 & 27-66 years old & Faith school & All Males \\
Cultural artist & 7 & - & 22-34 years old & Musical school, junior-senior high school & All Males \\
\end{tabular}


area.

Upon learning from informal leaders that most people in Mentawai attend church on Saturday to pray, the study identified a potential health communication channel. They asked the question, on what occasions do people mostly come.

\section{Almost all people come every Saturday to the church to pray (Mentawai District informal leader, 50).}

This finding supports the need of an approach development for community outreach in remote areas of Mentawai. People in the Mentawai District speak various languages, and most members of the community practice different religions. Hence a religious leader played a huge role in the community, the researcher probed and asked them if they were willing to promote TB education. Religious leaders in Mentawai were willing to participate in promoting TB messages during their speeches. A collaboration model with community leaders and religious leaders needed to be developed to mobilize the community and communicate information about the TB program.

Solok, meanwhile, has a different geography and cultural characteristics than Mentawai. Solok's informal leaders mentioned that people come to the village, mostly for parties since Saluang traditional music and storytelling are performed. They asked them on what occasions do people mostly come.
People mostly come if there is a party in the village. There will be a performance of Randai and Saluang traditional music (Solok District TB officer).

They meet and talk with each other at the fiesta. Therefore, they could use this existing channel to develop an approach for community outreach in remote areas. Traditional musicians were willing to promote TB messages during their performances by modifying their songs. The lyrics are mostly based on Minangkabau legends and folktales, so that they contained information aimed at reducing TB stigma in the community.

The study found that Knowledge-Attitude-Practice (KAP) and TB awareness of TB were barriers. Table 2 provides some examples of questions, meaning units, codes, categories, and sub-themes used in the analysis. The theme of reaching hard-to-reach populations in TB programs showed that specific health communication channels are appropriate for community outreach to increase TB knowledge and awareness, especially for people living in remote areas. The study's findings suggested areas of intersection between patients' health care and their cultural-religious beliefs that need a unique health communication channel in hard-to-reach populations. This theme indicated problems in TB control programs and the need for a channel targeting hard-to-reach populations.

The sub-theme of problems in TB control programs

Table 2. Derivation of themes: Examples of Questions, Meaning Units, Codes, Categories, and Sub-themes

\begin{tabular}{|c|c|c|c|c|}
\hline Questions & Meaning Unit & Codes & Category & Sub-theme \\
\hline \multirow[t]{4}{*}{$\begin{array}{l}\text { What is the experience } \\
\text { gained in TB control }\end{array}$} & $\begin{array}{l}\text { TB is still stigmatized, and knowledge about the disease } \\
\text { and treatment remains low (informant } 45 \text {, TB officer) }\end{array}$ & $\begin{array}{l}\text { Knowledge, Attitudes, } \\
\text { Practices (KAP) }\end{array}$ & $\begin{array}{l}\text { KAP and awareness } \\
\text { of TB are low due to } \\
\text { stigma, which impacts } \\
\text { TB coverage }\end{array}$ & \multirow[t]{4}{*}{$\begin{array}{l}\text { A problem in the } \\
\text { TB control program }\end{array}$} \\
\hline & $\begin{array}{l}\text { Due to the diversity found in TB's clinical manifestations, } \\
\text { not all patients in the community are recorded by health- } \\
\text { care providers. Generally, only patients with severe mani- } \\
\text { festations are likely to be recorded by the healthcare } \\
\text { provider, whereas those without apparent clinical manifes- } \\
\text { tations are not (informant 35, a medical doctor in primary } \\
\text { health care) }\end{array}$ & Case detection rate & & \\
\hline & $\begin{array}{l}\text { There is no comprehensive and coordinated approach to } \\
\text { engage the community in supporting TB services, includ- } \\
\text { ing helping patients continue treatment or proactively iden- } \\
\text { tifying possible TB cases (Informant } 50 \text {, TB officer) }\end{array}$ & $\begin{array}{l}\text { Community engagement, } \\
\text { awareness }\end{array}$ & $\begin{array}{l}\text { The system of } \mathrm{TB} \\
\text { control in the com- } \\
\text { munity has not been } \\
\text { planned optimally }\end{array}$ & \\
\hline & $\begin{array}{l}\text { We have a broad and remote area in which to reach TB } \\
\text { patients. Sometimes some landslides and storms isolate } \\
\text { the area (informant } 34, \mathrm{~TB} \text { officer) }\end{array}$ & Access & Hard-to-reach population & \\
\hline $\begin{array}{l}\text { On what occasions are } \\
\text { people mostly coming }\end{array}$ & $\begin{array}{l}\text { Mentawai: } \\
\text { Almost all people come every Saturday to the church to } \\
\text { pray (informant } 56 \text {, informal leader) } \\
\text { Solok: } \\
\text { People mostly come if there is a party in the village. } \\
\text { There will be a performance of Saluang (traditional music). } \\
\text { They will meet each other, have fun (informant } 60 \text {, chief } \\
\text { of the village) }\end{array}$ & $\begin{array}{l}\text { Religious leader approach } \\
\text { in Mentawai and the }\end{array}$ & $\begin{array}{l}\text { Different channels to reach } \\
\text { hard-to-reach populations } \\
\text { in different regions }\end{array}$ & $\begin{array}{l}\text { Channel to reach } \\
\text { the hard-to- } \\
\text { reach population }\end{array}$ \\
\hline
\end{tabular}

Notes: TB: Tuberculosis; KAP: Knowledge, Attitude, and Practice 
consisted of three categories: (1) KAP and awareness of TB is low, affecting TB coverage; (2) the community system of TB control has not been planned optimally; and (3) the problem of hard-to-reach populations. The subtheme of a channel for hard-to-reach populations consisted of one category: the need for different channels to reach hard-to-reach populations in different regions.

\section{Discussion}

The present study demonstrates that local leaders and musicians could actively participate as a health communication channel for community outreach. This communication was included in vulnerable populations by delivering TB education during religious community meetings, traditional culture meetings, and musical events that attract hard-to-reach people.

The involvement in health communication of religious and informal leaders and village community cadres strengthened the TB control program's ability to network with stakeholders and community groups. These findings are similar to those of Pirkani, et al., ${ }^{13}$ In their study, religious leaders played a role in increasing TB CDRs in Balochistan, Pakistan. Also, in Africa, faith healers could be more widely utilized in HIV/TB prevention programs as risk reduction counselors, in particular, on matters of community-level education. ${ }^{14}$

This study was in line with the recent Rio Political Declaration on Social Determinants of Health, adopted at the World Conference on Social Determinants of Health, which expresses a commitment to comprehensive intersectoral approaches that address health's social determinants and well-being to achieve social and health equity. This focus suggests three main actions relevant to TB control in Indonesia: (1) involvement of organized communities and all levels of government (local, provincial, and national); (2) attention to the fact that solutions often lie beyond the health sector, and require the engagement of many different sectors of government and society; and (3) recognition that local leaders and governments can and should play a key role. ${ }^{2}$

The role of religious leaders was leveraged to produce an impact on diseases other than TB. In Uganda, faithbased initiatives involving religious leaders and organizations have impacted HIV/AIDS prevention. In a project focused on communicating information about measles vaccination to hard-to-reach individuals, Minetti, et al., 8 found that addressing spiritual issues could make a big difference in an individual's illness experienceand often in health outcomes as well. The present study demonstrates make an insight that putting spirituality into medical education has become a priority among integrative medicine leaders, recognizing that attention to spirituality is also significant for healthcare providers themselve.
The cultural context also played a part in health communication, 12 establishing the role of local culture such as Saluang in raising TB awareness among the general population. Traditional musicians incorporated TB-related messages to improve awareness of the signs and symptoms of $\mathrm{TB}$ and knowledge about available services to encourage health-seeking behavior. It was attractive to the community because the held events were entertaining while providing information about TB.

Some related studies on the role of cultural content in health communication found that developing a culturally sensitive educational intervention program could reduce TB's high incidence. Furthermore, such efforts could play an essential role in reducing the stigma associated with the disease. ${ }^{6,10,11,14}$

It is thought that community health campaigns that incorporate cultural approaches may significantly impact TB control. Given that health decision-making is often a communal rather than an individual process in many lowincome countries, including Indonesia, community health education through cultural approaches seems warranted. ${ }^{14}$

Study in another setting has shown the need to address cultural health beliefs about the locus of control in designing and developing TB care and treatment programs. ${ }^{11,12}$ The study results revealed that foreign-born Vietnamese clients tended to operate with an internal locus of control, to attribute the resolution of health problems, such as TB, to chance rather than something that could be controlled by others. A controlled study of an HIV/AIDS/STI/TB intervention with faith healers in Vhembe District, South Africa, also mentions faith healers' role. ${ }^{15}$ They could be more widely utilized in HIV/AIDS/STI/TB prevention programs as risk reduction counselors, particularly on community-level education matters.

The present study identified gaps in knowledge about the cause of TB, inappropriate healthcare-seeking behavior, and stigma toward TB. There is the role of stigma in health promotion and disease control. ${ }^{15}$ The stigma that accompanies TB can harm the individual and family and result in their withdrawal from society because of shame and fear. This result highlights the need for education to provide practical strategies for individuals and families and teach communities where tuberculosis is endemic. 5,15

In other studies, traditional beliefs, such as the "evil eye", Satan, and witchcraft, were the most common perceived causes of TB. 3,5 In Tanzania and India, just like in West Sumatera, many people also mentioned that witchcraft could be the cause of TB. ${ }^{3,4}$ Studies in India mention that concealment of disease was explained as the fear of losing social status, marital problems, and hurtful behavior by the community. ${ }^{4}$ These traditional beliefs 
might contribute to TB's spread, as people with such beliefs may not visit health facilities.

Discovering the broader context of a patient's life may be critical to providing responsive care and assuring good TB health outcomes. ${ }^{13}$ Such approaches may increase awareness, knowledge, CDRs, access to TB treatment, treatment adherence, and successful treatment.

Another lesson learned from our study was that the initial engagement should be tailored to enhance message relevance. It is necessary to identify situations where such messages can be communicated. We had to tailor messages to be distributed in the outreach community. This study implies that healthcare providers may sometimes be uncomfortable talking about cultural-religious health beliefs and behaviors with patients and families. As a community health literacy problem, this condition becomes a challenge for health officers promoting TB prevention, diagnosis, and care. The need for adequate language interpreters in healthcare settings has been uniformly addressed. However, it is also imperative for people to communicate with leaders of their faith community. These influential figures can help patients and families interpret what is happening on a spiritual level during a health crisis. Religious leaders can clarify which tenets cut across the branches of their faith in matters related to TB. ${ }^{13}$ Healthcare providers should tailor health communication to enhance message relevance and should have the skills to identify health communication situations. Different channels are needed to reach hard-to-reach populations in different regions.

\section{Conclusion}

This study has identified cultural and religious belief approaches that play significant roles in the Tuberculosis Program for hard-to-reach populations in Mentawai and Solok West Sumatera-Indonesia. Specific health communication channels were needed in different regions based on their different cultures and beliefs.

This study suggested that the initial engagement should be tailored to enhance message relevance. Besides, healthcare providers must identify situations where such messages can be communicated. These customized messages need to be distributed for successful the outreach community.

\section{Abbreviations}

TB: Tuberculosis; CDRs: Case Detection Rates; KAP: Knowledge, Attitudes, and Practices.

\section{Ethics Approval and Consent to Participate}

Ethical approval for the study was obtained from the Faculty of Medicine's ethics committee at Andalas University, Indonesia, with decree number: $127 / \mathrm{KEP} / \mathrm{FK} / 2015$. Participation was voluntary, and the participants provided their consent to participate. All the interviews were audiotaped with the consent of the participants. The participants were assured that their information would remain confidential, and they had the right to withdraw from the study at any time. Anonymity was maintained during data analysis and presentation.

\section{Competing Interest}

Author declares that there are no significant competing financial, professional, or personal interests that might have affected the performance or presentation of the work described in this manuscript.

\section{Availability of Data and Materials}

The datasets generated during and/or analyzed during the current study are available from the corresponding author on reasonable request.

\section{Authors' Contribution}

RM designed the study; RM, IM, and FFY collected the data and contributed to data analysis, interpretation of findings, and drafting the manuscript. All the authors read and approved the final manuscript.

\section{Acknowledgment}

This work would not have been possible without the support of Jaringan Kesehatan/Kesejahteraan Masyarakat (JKM), Networking for Community Welfare and Health JKM, Westat, USAID, the district health offices of Mentawai and Solok, and the Community Empowerment Against Tuberculosis (CEPAT). The authors would like to thank all the team for supporting the implementation and monitoring of the project and collecting and analyzing the data. Thanks to Professor Nawi $\mathrm{Ng}$, who facilitated in preparing and finalizing this manuscript.

\section{References}

1. Indonesia Ministry of Health. National Tuberculosis program: current status of integrated community based TB service delivery and the Global Fund work plan to find missing TB cases; 2015.

2. World Health Organization. Global tuberculosis report 2015. 20th Ed. Geneva: World Health Organization; 2015.

3. Abebe G, Deribew A, Apers L, Woldemichael K, Shiffa J, Tesfaye M, et al. Knowledge, health-seeking behavior and perceived stigma towards tuberculosis among tuberculosis suspects in a rural community in southwest Ethiopia. PLoS One. 2010; 5 (10): e13339.

4. Atre S, Kudale A, Morankar S, Gosoniu D, Weiss M. Gender and community views of stigma and tuberculosis in rural Maharashtra, India. Global Public Health. 2011; 6 (1): 56-71.

5. Juniarti N, Evans D. A qualitative review: the stigma of tuberculosis. Journal of Clinical Nursing. 2011; 20 (13-14): 1961-70.

6. Jit M, Stagg HR, Aldridge RW, White PJ, Abubakar I. Dedicated outreach service for hard to reach patients with tuberculosis in London: an observational study and economic evaluation. BMJ. 2011; 343: d5376.

7. Lu S, Tian B, Kang X, Zhang W, Meng X, Zhang J, et al. Public awareness of Tuberculosis in China: a national survey of 69,253 subjects. The International Journal of Tuberculosis and Lung Disease. 2009; 13 (12): 1493-9.

8. Minetti A, Hurtado N, Grais RF, Ferrari M. Reaching hard-to-reach individuals: nonselective versus targeted outbreak response vaccination 
for measles. American Journal of Epidemiology. 2014; 179 (2): 24551.

9. Vukovic DS, Nagorni-Obradovic LM. Knowledge and awareness of tuberculosis among the Roma population in Belgrade: a qualitative study. BMC Infectious Diseases. 2011; 11 (1): 284.

10. Haakenstad A, Johnson E, Graves C, Olivier J, Duff J, Dieleman JL. Estimating the development assistance for health provided to faithbased organizations, 1990-2013. PLoS One. 2015; 10 (6): e0128389.

11. Olivier J, Tsimpo C, Gemignani R, Shojo M, Coulombe H, Dimmock $\mathrm{F}$, et al. Understanding the roles of faith-based healthcare providers in Africa: review of the evidence with a focus on magnitude, reach, cost, and satisfaction. The Lancet. 2015; 386 (10005): 1765-75.

12. Mashamba T, Peltzer K, Maluleke TX, Sodi T. A controlled study of an HIV/AIDS/STI/TB intervention with faith healers in Vhembe
District, South Africa. African Journal of Traditional, Complementary, and Alternative Medicines. 2011; 8 (5 Suppl): 83-9.

13. Wiley AS, Allen JS. Medical anthropology: a biocultural approach. Third Edition. University Press Oxford; 2009.

14. Mason PH, Roy A, Spillane J, Singh P. Social, historical and cultural dimensions of tuberculosis. Journal of Biosocial Science. 2016; 48: 206-32.

15. Chang S, Cataldo J. A systematic review of global cultural variations in knowledge, attitudes, and health responses to tuberculosis stigma. The International Journal of Tuberculosis and Lung Disease. 2014; 18 (2): $168-73$. 